\title{
Nab-paclitaxel in first-line treatment of advanced non-small-cell lung cancer
}

See Commentary on page 164

$\mathrm{N}$ anoparticle albumin-bound (nab-) paclitaxel is a solvent-free paclitaxel formulation that has been designed to reduce adverse reactions associated with conventional solvent-based paclitaxel formulations and to improve paclitaxel tumor penetration by exploiting the physiologic transport properties of albumin. In a recently reported phase 3 trial that compared nab-paclitaxel and solvent-based paclitaxel injection in combination with carboplatin as first-line treatment of advanced nonsmall-cell lung cancer (NSCLC), nab-paclitaxel was associated with a significantly greater overall response rate (ORR), the primary end point, and a reduced risk of neuropathy. ${ }^{1}$ The findings in this international trial, combined with the demonstration of paclitaxel efficacy in this setting, supported the recent approval of nab-paclitaxel combined with carboplatin as first-line treatment of advanced NSCLC. Subset analyses in the trial suggested some potential response and survival advantages with nab-paclitaxel treatment.

Of 1,052 patients with nonresectable stage $3 \mathrm{~B}$ or stage 4 NSCLC, 521 received a weekly nab-paclitaxel 100 $\mathrm{mg} / \mathrm{m}^{2}$ infusion and 531 received conventional solventbased paclitaxel $200 \mathrm{mg} / \mathrm{m}^{2}$ every 3 weeks. All of the patients received carboplatin at area under the concentrationtime curve (AUC) 6 once every 3 weeks. Steroid/antihistamine premedication was required in the solvent-based paclitaxel group and was used at investigator discretion in the nab-paclitaxel group. Patients were to receive at least 6 cycles of treatment. The primary endpoint was ORR.

The median age of the patients was 60 years in the nab-paclitaxel and solvent-based paclitaxel groups, with $14 \%$ and $15 \%$ of patients, respectively, being 70 years or older; $75 \%$ of patients in both groups were men, and $80 \%$ and $82 \%$ were white. Global geographic distribution was balanced, with most patients being from Russia (46\% and 44\%) and Ukraine (23\% and 25\%), followed by Japan (14\% and 14\%) and the United States (12\% and 11\%). ECOG performance status was 1 in $74 \%$ of the nabpaclitaxel group, and $78 \%$ of the solvent-based paclitaxel group. Histology consisted of adenocarcinoma in 49\% and $50 \%$ of patients, and squamous cell carcinoma in $44 \%$ and $42 \%$; and $79 \%$ of each group had stage IV disease. In

Report prepared by Matt Stenger, MS

\section{What's new, what's important}

The Food and Drug administration has approved nab-paclitaxel plus carboplatin for patients with untreated locally advanced or metastatic non-smallcell lung cancer who are not candidates for surgery or radiation. The approval was based on results from the phase $3 \mathrm{CA} 031$ trial, which showed that weekly nab-paclitaxel, a nanoparticle albuminbound formulation of paclitaxel, combined with carboplatin significantly improved overall response rate (ORR), when compared with solvent-based paclitaxel plus carboplatin. All of the responses were partial, except for 1 complete response in the solventbased paclitaxel group. ORR was significantly greater with nab-paclitaxel among patients with squamous cell histology ( $41 \%$ vs $24 \%$, respectively; RRR, 1.680 ; $P<.001$ ), with no difference between treatments being observed in patients with nonsquamous histology (ORR, $26 \%$ vs $25 \%$ ) or adenocarcinoma (ORR, $26 \%$ vs $27 \%$ ).

Grade 3 and 4 thrombocytopenia and anemia were significantly more common in nab-paclitaxel patients than in solvent-based paclitaxel patients, but grade 3 and 4 neutropenia was significantly more common in solvent-based paclitaxel patients than in nab-paclitaxel patients, as were all grades of sensory neuropathy, grades 3 and 4 neuropathy, grade 3 myalgia and arthralgia. A subsequent analysis of a subset of patients 70 years or older reported that survival for the nabpaclitaxel arm was significantly longer in those $\mathrm{pa}^{-}$ tients (median OS, 19.9 vs 10.4 months), but it is not clear what that benefit is due to. Fewer side effects, better tolerability, and improved response rates are seen with nab-paclitaxel-containing regimen. That is a promising step forward and it could potentially help many patients. But lack of survival benefit is disappointing. In the era of molecular classification and targeting, this may not be the most exciting development in lung cancer treatment.

- Jame Abraham, MD 


\section{How I treat advanced NSCLC}

Non-small-cell lung cancer frequently presents at an advanced stage. In this setting, chemotherapy has been shown not only to improve overall survival, but also quality of life. Platinum-based chemotherapy remains the mainstay of treatment for metastatic disease. Treatment of patients with advanced NSCLC usually begins with review of histology, which is an important factor for individualizing treatment. The use of bevacizumab and pemetrexed is restricted to patients with nonsquamous cell NSCLC. Superior treatment outcomes with pemetrexed in nonsquamous NSCLC and association of severe pulmonary hemorrhage with the use of bevacizumab in squamous NSCLC have led to these limitations. There are, as a consequence, fewer active agents for patients with squamous cell NSCLC compared with adenocarcinoma.

Nab-paclitaxel, which was recently approved for use as a first-line agent in the treatment of NSCLC showed higher response rate in squamous NSCLC, and improved overall survival in elderly patients, and represents an option for these subsets of patients. Advances in molecular characterization of NSCLC have made per- sonalized approaches feasible. For patients with adenocarcinoma, especially never smokers and for those without significant smoking history, molecular testing is routinely used to guide therapeutic decisions. Detection of gain of function mutations in the epidermal growth factor receptor domain and ALK gene rearrangements select patients who benefit from the use of erlotinib and crizotinib respectively.

Maintenance therapy has also recently emerged as a treatment paradigm for advanced NSCLC. Continuation maintenance, where a part of initial chemotherapy is continued, and switch maintenance, where a new non-cross-resistant agent is introduced after completion of first-line therapy have been studied. As we gain a better understanding of the biology of NSCLC, patients should be offered enrollment and treatment as part of clinical trial. Several new treatments that irreversibly target EGFR, novel EML4-ALK inhibitors, and agents that target KRAS mutations are in development with encouraging early efficacy data.

- Charu Aggarwal, MD all, $26 \%$ and $27 \%$ of patients had never smoked, $41 \%$ and $44 \%$ were still smoking at the time of the study. Prior therapy included radiation therapy in $7 \%$ of nab-paclitaxel patients and $9 \%$ of solvent-based paclitaxel patients, and chemotherapy in $3 \%$ and $2 \%$, respectively.

Patients in both groups received a median of 6 cycles of treatment. On independent radiology assessment, the ORR was $33 \%$ in the nab-paclitaxel group, compared with $25 \%$ in the solvent-based paclitaxel group (response rate ratio [RRR], $1.313 ; 95 \% \mathrm{CI}, 1.082-1.593 ; P=.005)$. All of the responses were partial, except for 1 complete response in the solvent-based paclitaxel group. ORR was significantly greater with nab-paclitaxel among patients with squamous cell histology ( $41 \%$ vs $24 \%$; RRR, $1.680 ; P<.001$ ), with no difference between treatments being observed in patients with nonsquamous histology (ORR, $26 \%$ vs $25 \%$ ) or adenocarcinoma (ORR, $26 \%$ vs $27 \%$ ).

There nab-paclitaxel group had nonsignificant 10\% improvement in median progression-free survival (PFS, 6.3 vs 5.8 months; hazard ratio [HR], 0.902; 95\% CI, 0.7671.060 ) and a nonsignifant $8 \%$ improvement in median overall survival (OS, 12.1 vs 11.2 months; HR, 0.922; 95\% CI, 0.797-1.066). The overall PFS and OS comparisons met criteria for noninferiority of nab-paclitaxel.
Subgroup analyses suggested potential differences in survival according to geographic region and age. Median PFS was nonsignificantly prolonged with nab-paclitaxel treatment among the 165 patients from the US and Canada (7.0 vs 5.4 months; HR, 0.694) and among patients aged 70 years or older ( 8.0 vs 6.8 months; HR, 0.687 ). Median OS was significantly prolonged with nabpaclitaxel treatment in North American patients (12.7 vs 9.8 months; HR, $0.622 ; P=.008)$ and in patients aged $\geq 70$ years (19.9 vs 10.4 months; HR, $0.583 ; P=.009$ ). No differences between treatments were observed among 724 patients from Russia and Ukraine, among $149 \mathrm{pa}^{-}$ tients from Japan, or among patients younger than 70 years. No significant differences in median OS were found between nab-paclitaxel and solvent-based paclitaxel patients with squamous histology (10.7 vs 9.5 months) or nonsquamous histology (13.1 vs 13.0 months).

Second-line therapy was used in 53\% of the nabpaclitaxel group and in 54\% of the solvent-based paclitaxel group. Use of second-line therapy was most common in Japan (85\%), Australia (79\%), and North America (69\%) and least common in Russia and Ukraine (44\%). As in the analysis in the overall population, the nab-paclitaxel group had a nonsignificant increase in OS among patients who received second-line therapy. 
Safety data were reported as treatment-related adverse events. Grade 3 and 4 thrombocytopenia was significantly more common in nab-paclitaxel patients than in solventbased paclitaxel patients (grade 3,13\% and $5 \%$ vs grade 4 , $7 \%$ and $2 \%$, respectively; $P<.001$ ) as was anemia (grade 3, $22 \%$ and $5 \%$ vs grade $4,6 \%$ and $<1 \% ; P<.001$ ). Grade 3 and 4 neutropenia was significantly more common in solvent-based paclitaxel patients than in nab-paclitaxel $\mathrm{pa}^{-}$ tients (grade $3,32 \%$ and $26 \%$ vs grade $4,33 \%$ and $14 \% ; P<$ .001). Febrile neutropenia occurred in $1 \%$ of both groups. Sensory neuropathy of all grades was significantly more common with solvent-based paclitaxel (62\% vs $46 \%$; $P<.001)$, as was grade 3 and 4 neuropathy (11\% and $<1 \%$ vs $3 \%$ and $0 \% ; P<.001)$. The median time to improvement of grade 3 or 4 sensory neuropathy to grade 1 was 38 days in the nab-paclitaxel group and 104 days in the solvent-based paclitaxel group. Grade 3 myalgia was more common with solvent-based paclitaxel ( $2 \%$ vs $<1 \% ; P=.011)$ as was grade 3 arthralgia ( $2 \%$ vs $0 \% ; P=.008)$. One treatmentrelated death occurred in each group.

Treatment was discontinued because of unacceptable toxicity without progressive disease in $12 \%$ of both groups, and because of adverse events in $4 \%$ of nab- paclitaxel patients and 5\% of solvent-based paclitaxel patients. Paclitaxel dose reductions occurred in $46 \%$ of nabpaclitaxel patients and $23 \%$ of solvent-based paclitaxel patients, with reductions occurring primarily as a result of neutropenia ( $29 \%$ vs $10 \%)$, thrombocytopenia ( $13 \%$ vs $4 \%)$, anemia ( $6 \%$ vs $<1 \%$ ), and sensory neuropathy $(2 \%$ vs $6 \%)$. Overall, paclitaxel dose intensity was $26 \%$ greater and the cumulative paclitaxel dose was $18 \%$ greater in the nab-paclitaxel group. Dose delays occurred in $82 \%$ of nab-paclitaxel patients and in 54\% of solvent-based paclitaxel patients.

Patients were assessed with the FACT-Taxane scale on day 1 of each treatment cycle. The nab-paclitaxel group exhibited a significantly greater improvement in mean change from baseline to final evaluation on this scale compared with the solvent-based paclitaxel group, including improvements on the neuropathy $(P<.001)$, pain $(P<.001)$, and hearing loss $(P=.002)$ subscales.

\section{Reference}

1. Socinski MA, Bondarenko I, Karaseva NA, et al. Weekly nabpaclitaxel in combination with carboplatin versus solvent-based paclitaxel plus carboplatin as first-line therapy in patients with advanced non-small-cell lung cancer: final results of a phase III trial. J Clin Oncol. 2012;30:2055-2062. 